\title{
Signal Separation in Ultrasonic Non-Destructive Testing
}

\author{
V. Matz, M. Kreidl, R. Šmíd
}

In ultrasonic non-destructive testing the signals characterizing the material structure are commonly evaluated. The sensitivity and resolution of ultrasonic systems is limited by the backscattering and electronic noise level commonly contained in the acquired ultrasonic signals. For this reason, it is very important to use appropriate advanced signal processing methods for noise reduction and signal separation. This paper compares algorithms used for efficient noise reduction in ultrasonic signals in A-scan. Algorithms based on the discrete wavelet transform and the Wiener filter are considered. Part of this paper analyses and applies blind source separation, which has never been used in practical ultrasonic non-destructive testing. All proposed methods are evaluated on both simulated and acquired ultrasonic signals.

Keywords: ultrasonic testing, de-noising algorithms, noise reduction

\section{Introduction}

Ultrasonic non-destructive testing is commonly used for flaw detection in materials. Ultrasound uses the transmission of high-frequency sound waves in a material to detect a discontinuity or to locate changes in material properties. Ultrasonic wave propagation in the tested materials is essentially influenced by the structure of the material. Due to the material structure, the acquired ultrasonic signal can be corrupted by a relatively high noise level, commonly called backscattering noise. Another source of noise is from the electronic circuitry. These noise components are generally present in all acquired ultrasonic signals, together with flaw and back-wall echo. Back-wall echo is due to the reflection of an ultrasonic wave from the end of the material, and fault echo is caused by the reflection of ultrasonic waves from cracks or defects.

The main task here is to detect the fault echo in an ultrasonic signal; i.e., to locate the cracks or defects in the tested materials. The flaw detection efficiency is mainly influenced by the noise level (backscattering and electronic), and for this purpose efficient signal processing techniques used for noise reduction and signal separation are proposed. In the past, many methods have been evaluated [2-5, 11] for efficient noise reduction in ultrasonic signals. The simplest method [2] is based on averaging the acquired ultrasonic signals. Other popular methods are based on filters [2] with finite (FIR) and infinite impulse response (IIR). These methods are quite simple, but the noise suppression is not effective. Non-linear methods based on band-pass filters, known as split spectrum processing, offer greater signal-to-noise improvement, but the setting of the parameters is based on heuristic methods, with varying results. A very popular method is based on the discrete wavelet transform algorithm $[3,6,7]$. This method is very efficient, but it is important to choose the proper mother wavelet, threshold level and threshold rule [8]. Other methods used for signal de-noising are based on adaptive algorithms derived from the Wiener filter [9, 10].

This paper presents and evaluates methods used for ultrasonic signal de-noising: the discrete wavelet transform, the Wiener filter and blind source separation with appropriate settings. These methods with selected parameters are evaluated in terms of signal-to-noise improvement and flaw detection efficiency. Another method used for ultrasonic signal and noise separation is also proposed, and its applicability is discussed in detail. This method is based on independent component analysis, and it has never been applied before in the ultrasonic non-destructive testing area.

The rest of this paper is structured as follows. The second section offers basic theoretical descriptions of the de-noising and signal separation methods. In the third section there is an evaluation of methods with different parameter settings. For the case of the blind signal separation method, the appropriate configuration of ultrasonic transducers is described. Based on the theoretical analysis, all methods are applied to real acquired ultrasonic signals in section four. For the evaluation, samples of materials used for constructing aircraft engines were used. Finally, the results are discussed and future work is indicated.

\section{De-noising and signal separation methods}

\subsection{Discrete wavelet transform}

The wavelet transform [3, 6, 7] is a multiresolution analysis technique that can be used to obtain a time-frequency representation of an ultrasonic signal. The discrete wavelet transform (DWT) analyzes the signal by decomposing it into its coarse and detailed information, which is accomplished with the use of successive high-pass and low-pass filtering and subsampling operations, on the basis of the following equations:

$$
\begin{aligned}
& y_{\text {high }}(k)=\sum_{n} x(n) \cdot g(2 k-n), \\
& y_{\text {low }}(k)=\sum_{n} x(n) \cdot h(2 k-n),
\end{aligned}
$$

where $y_{\text {high }}(k)$ and $y_{\text {low }}(k)$ are the outputs of high-pass and low-pass filters with impulse response $g$ and $h$, respectively, after subsampling by 2 (decimation). This procedure is repeated for further decomposition of the low-pass filtered signals.

Starting from the approximation and detailed coefficients, the inverse discrete wavelet reconstructs the signal, 
inverting the decomposition step by inserting zeros and convolving the results with the reconstruction filters.

DWT can be used as an efficient de-noising method for families of signals that have a few nonzero wavelet coefficients for a given wavelet family. This is fulfilled for most ultrasonic signals. The common filtering procedure (also called de-noising) affects the signal in both frequency and amplitude, and involves three steps. The basic version of the procedure consists of:

a) decomposing the signal using DWT into $N$ levels using filtering and decimation to obtain the approximation and detailed coefficients,

b) thresholding the detailed coefficients,

c) reconstructing the signal from detailed and approximation coefficients using the inverse transform (IDWT).

When decomposing the signal it is important to choose a suitable mother wavelet, threshold rule and threshold level.

\subsection{Wiener filter based group delay statistics}

The Wiener filter $[9,10]$ is a global filter and produces an estimation of the uncorrupted signal by minimizing the mean square error between the estimated signal and the uncorrupted signal in a statistical sense. The process representing the received signal consists of signal and noise, both uncorrelated zero-mean wide-sense-stationary random processes.

By filtering $y(t)$ we estimate $s(t)$ using a time-invariant linear system with transfer function $H(f)$. The resulting mean-square error will then be

$e=\int_{-\infty}^{\infty}|1-H(f)|^{2} S(f) \mathrm{d} f+\int_{-\infty}^{\infty}|H(f)|^{2} N(f) \mathrm{d} f$,

where $N(f)$ and $S(f)$ are power spectral densities of the noise and the signal. Error $e$ is minimized over $H(f)$ for fixed $S(f)$ and $N(f)$. The transfer function can be estimated by means of the group delay target signal having a deterministic phase delay over the working frequency [9]. The following techniques are based on using a discrete group delay. It can be calculated by

$$
T(k)=-\frac{N}{2 \pi}[\phi(k+1)-\phi(k)],
$$

where $\phi(k)$ is the phase component of the discrete Fourier transform, $k$ is the frequency index and $N$ is the total number of points. To minimize the edge effect, various windows for the received time sequence are applied. To obviate discontinuity in the group delay phase unwrapping techniques are used. Two useful variants [9] based on group delay statistics are the group delay moving standard deviation

$$
\sigma_{k}=\left[\frac{1}{M-1} \sum_{m=k}^{M+k}\left\{\Delta T(m)-\Delta \bar{T}_{k}\right\}^{2}\right]^{\frac{1}{2}}
$$

and the group delay moving entropy

$$
H_{k}=-\sum_{j=k+1}^{M+k} f\left(T_{j}\right) \log _{2}\left[f\left(T_{j}\right)\right] .
$$

Both estimates are computed within a moving window $M$. Window $M$ is set to a small compared data length, and reflects a trade off between resolution and estimation error.

\subsection{Blind signal separation}

Blind signal separation (BSS) consists in recovering unobserved signals or sources from several observed mixtures. The simplest BSS model assumes the existence of $n$ independent signals $s_{1}(t), \ldots, s_{n}(t)$ and the observation of as many mixtures $x_{1}(t), \ldots, x_{n}(t)$, these mixtures being linear and instantaneous. This is compactly represented by the mixing equation

$$
x(t)=A s(t),
$$

where $\boldsymbol{s}(t)=\left[s_{1}(t), \ldots, s_{n}(t)\right]^{\mathrm{T}}$ is a column vector collecting the source signals, vector $x(t)$ similarly collects the $n$ observed signals and the square mixing matrix $\boldsymbol{A}$ contains the mixture coefficients. The BSS problem consists in recovering the source vector $\boldsymbol{s}(t)$ using only the observed data $x(t)$, the assumption of independence between the entries of the input vector $\boldsymbol{s}(t)$ and possibly some a priori information about the probability distribution of the inputs. This can be formulated as the computation of an $n \times n$ separating matrix $\boldsymbol{B}$ whose output $\boldsymbol{y}(t)$

$$
y(t)=\boldsymbol{B} \boldsymbol{x}(t)
$$

is an estimate of the vector $\boldsymbol{s}(t)$ of the source signals. The basic BSS model can be extended in several directions taking into account, for example more sensors than sources, noisy observations, complex signals and mixtures. The solution of equation (7) depends on the selected algorithm. Many algorithms have been published with different results. One of the

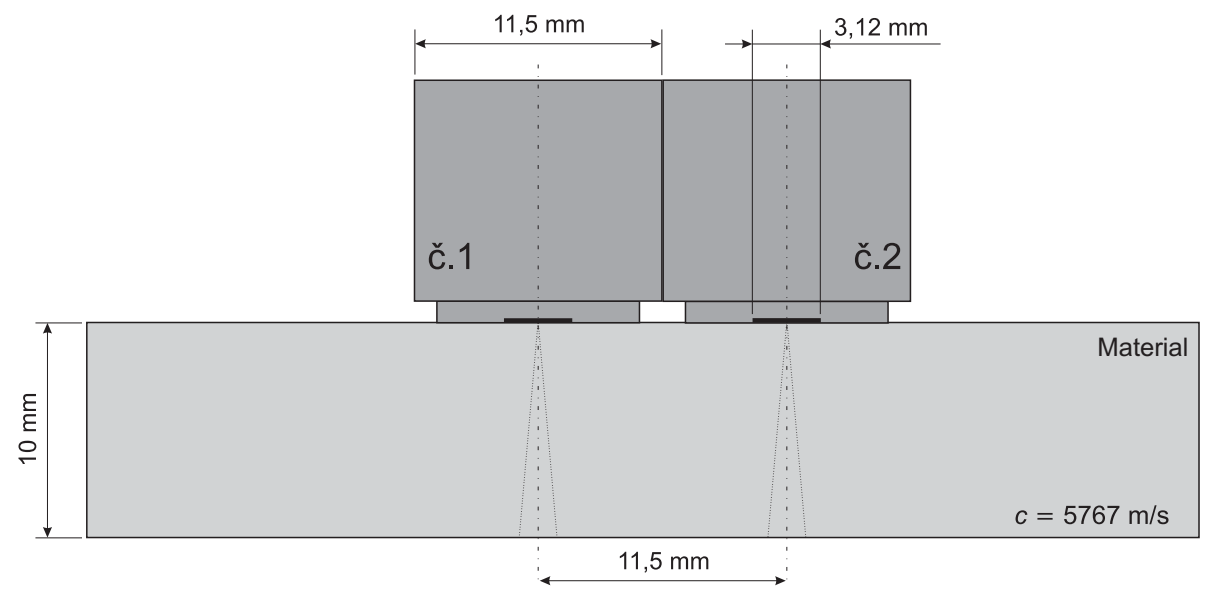

Fig. 1. Configuration of ultrasonic transducers for BSS 
most popular algorithms, called FastICA (Fast Independent Component Analysis), is described in detail in [12, 13].

Before the FastICA algorithm can be used, it is very important to characterize the model for the ultrasonic signal separation. The main question is how to propose the source signals $\boldsymbol{s}(t)$. In our study, we used two ultrasonic transducers and acquired the ultrasonic signals synchronously with the ultrasonic transducer configuration, as shown in Fig. 1.

\section{Theoretical results}

First of all, for the detailed analysis and for performing the de-noising methods it is necessary to generate the simulated ultrasonic signal. The signal is simulated based on the amplitude and frequency analysis of a set of acquired ultrasonic signals. Based on this analysis and a physical analysis of ultrasonic wave propagation, the signal was generated based on a simple clutter model using the equation [11]:

$H_{\text {mat }}(\omega)=$

$=\sum_{k=1}^{K_{\text {tot }}} \beta_{k} \frac{\omega^{2}}{x_{k}} \exp \left(-2 \alpha x_{k} \omega^{4}\right) \exp \left(-i \omega \frac{2 x_{k}}{c_{l}}\right) \cdot H(\omega) \cdot H(\omega)$,

where $\alpha$ is the material attenuation coefficient, $c_{l}$ is the velocity of the longitudinal waves, $x_{k}$ is the grain positions of $k=1 \ldots K_{\text {tot }}$ is the number of grains and $\beta_{k}$ is a random vector depending on the grain volume. An example of a generated ultrasonic signal is shown in Fig. 2. The signal consists of noise (backscattering, electronic), fault echo and back-wall echo.

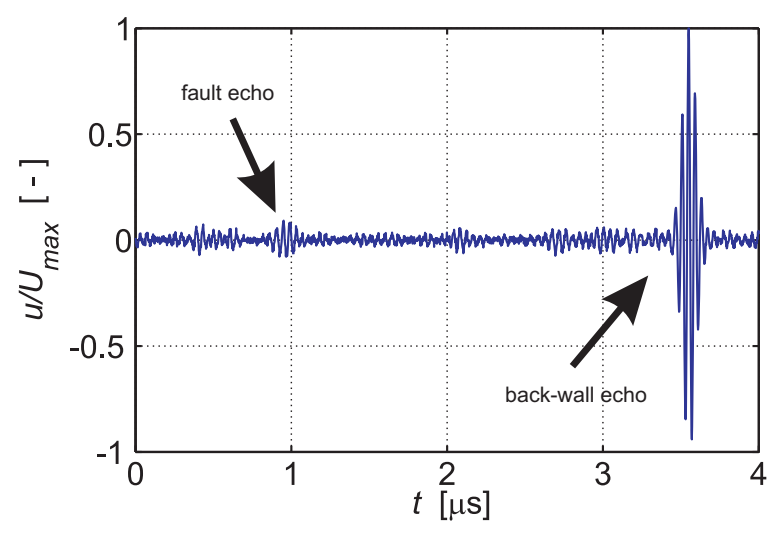

Fig. 2: Simulated ultrasonic signal

First of all, the DWT de-noising algorithm was used. For efficient noise reduction, it is necessary to select the shape of the mother wavelet, the threshold level and the threshold rule [8]. The shape of the mother wavelet has to be very similar to the ultrasonic echo [6]. It has to fulfill the following properties: symmetry, orthogonality and feasibility for DWT. A group of mother wavelets was tested: Haar's wavelet, the discrete Meyer wavelet, Daubechie's wavelet and Coiflet's wavelet. In the proposed procedure, only local thresholding of detailed coefficients was used. In the case of the thresholding rule, soft and hard thresholding can be used. According to the literature $[6,8]$, soft thresholding is not a proper option for noise reduction in ultrasonic signals, because the noise level and the amplitude of the fault echo are decreased by the threshold level. Other options for thresholding rules are to modify the hard thresholding rule using the following equations:

The compromise thresholding rule [7] can be defined as

$$
\hat{T}_{i j}=\left\{\begin{array}{c}
\operatorname{sign}\left(T_{i j}\right)\left(\left|T_{i j}\right|-a T\right),\left|T_{i j}\right| \geq T \\
0,\left|T_{i j}\right|<T
\end{array}\right.
$$

where $T_{i j}$ is the threshold level for sample $i$ at level $j$ and $\alpha$ is the coefficient for a compromise between hard and soft thresholding.

The custom thresholding rule [7] is defined as

$$
\hat{T}_{i j}=\left\{\begin{array}{c}
T_{i j}-\operatorname{sign}\left(T_{i j}\right)(1-\alpha) T,\left|T_{i j}\right| \geq T \\
0,\left|T_{i j}\right|<\tau \\
\alpha T\left(\frac{\left|T_{i j}\right|-\tau}{T-\tau}\right)^{2}\left\{(\alpha-3)\left(\frac{\left|T_{i j}\right|-\tau}{T-\tau}\right)+4-\alpha\right\}
\end{array}\right.
$$

where $\tau$ is the coefficient characterizing the sample level from which the thresholding is valid. The principle of compromising and custom thresholding together with hard and soft thresholding is shown in Fig. 3.

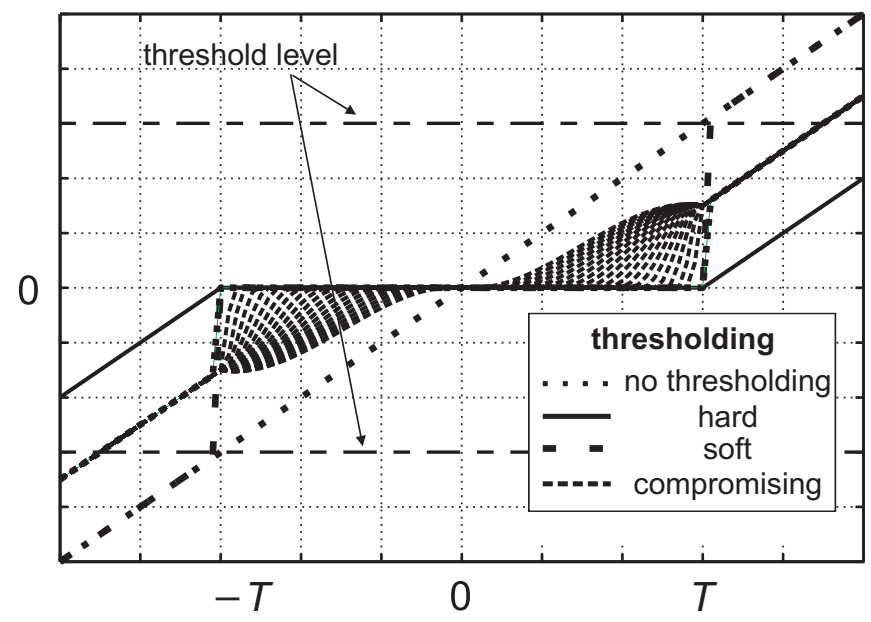

Fig. 3: Wavelet thresholding demonstration

We evaluated common thresholding methods implemented in the Matlab Wavelet toolbox [7] (rigsure, sqtwolog, heursure, minimaxi). Due to the unsatisfactory results we proposed a new method based on standard deviation $V_{1}$ and mean value together with standard deviation $V_{2}$. The local thresholds at each level of decomposition are given by

$$
V_{1}=\sqrt[k]{\frac{1}{n-1} \sum_{j=1}^{n}\left(c D_{j}-\overline{\boldsymbol{c D}}\right)^{2}}
$$

and

$$
V_{2}^{k}=\sqrt{\left(\mu+V_{1}\right)}
$$

where $n$ is the length of the vector detail coefficients, $k$ is a constant (crest factor), $\boldsymbol{c D}$ is the vector of detailed coefficients, and $\mu$ is the mean value. 
With the use of all the mother wavelets, proposed threshold levels and rules we evaluated the de-noising process on the simulated ultrasonic signals (Fig. 1) by calculating of two parameters. The first parameter evaluates the signal-to-noise ratio enhancement and can be expressed as

$$
S N R E=10 \log \frac{P_{1}}{P_{2}},
$$

where $P_{1}$ and $P_{2}$ are the power of the noise before and after de-noising. Another parameter evaluates the fault echo changes and the decrease the amplitude, and can be expressed as

$$
K c=R_{A_{a} A_{b}}(0)\left(1-\frac{A_{a}-A_{b}}{A_{b}}\right),
$$

where $R$ is the cross-correlation function, and $A_{b}$ and $A_{a}$ are the fault echo amplitudes before and after de-noising. Many combinations have been processed with different threshold levels, threshold rules and mother wavelets, and the fault echo within 1-100\% of initial echo amplitude was added to the simulated ultrasonic signal. In the case of threshold rule evaluation, parameters $k, \alpha$ and $\tau$ were changed within the appropriate range.

Best results for hard, custom and soft thresholding are shown in Table 1, Table 2 and Table 3.

It can be seen from Table 1 that in the case of hard thresholding the best results were obtained using the discrete Meyer mother wavelet and threshold level based on standard deviation. The value $S N R E=37.59 \mathrm{~dB}$ and fault echo with amplitude of the $5 \%$ of the initial echo amplitude was detected. Other thresholding rules and mother wavelets do not provide better results. The noise was suppressed and fault echo equal in amplitude to the noise level was efficiently detected.

The next method to be evaluated here is based on the Wiener filter, using group delay statistics. The Wiener filter based group delay moving entropy and group delay moving

Table 1: Evaluation of hard thresholding

\begin{tabular}{|l|c|c|c|c|c|c|c|c|}
\hline threshold level & \multicolumn{4}{|c|}{$V_{1}$} & \multicolumn{4}{c|}{$V_{2}$} \\
\hline mother wavelet / parameter & $\mathrm{db} 2$ & $\mathbf{d b 4}$ & $\mathrm{db} 6$ & $\mathbf{d m e y}$ & $\mathrm{db} 2$ & $\mathrm{db} 4$ & $\mathrm{db} 6$ & $\mathrm{dmey}$ \\
\hline $\max . D_{x}(-)$ & 0.994 & $\mathbf{0 . 9 8 9}$ & 0.978 & $\mathbf{0 . 9 8 1}$ & 0.967 & 0.976 & 0.966 & 0.984 \\
\hline $\max . S N R E(\mathrm{~dB})$ & 25.97 & $\mathbf{3 7 . 7 6}$ & 35.18 & $\mathbf{3 7 . 5 9}$ & 24.70 & 24.59 & 19.33 & 19.72 \\
\hline $\min . A_{f}(\%)$ & 9 & $\mathbf{7}$ & 9 & $\mathbf{5}$ & 13 & 9 & 20 & 2 \\
\hline $\min . k(-)$ & 1.35 & $\mathbf{2}$ & 1.1 & $\mathbf{1 . 4}$ & 1.35 & 4.5 & 1.4 & 1.4 \\
\hline
\end{tabular}

Table 2: Evaluation of compromise thresholding

\begin{tabular}{|l|c|c|c|c|c|c|c|c|}
\hline threshold level & \multicolumn{4}{|c|}{$V_{1}$} & \multicolumn{4}{c|}{$V_{2}$} \\
\hline mother wavelet / parameter & $\mathrm{db} 2$ & $\mathbf{d b 4}$ & $\mathrm{db} 6$ & $\mathbf{d m e y}$ & $\mathrm{db} 2$ & $\mathbf{d b 4}$ & $\mathrm{db} 6$ & dmey \\
\hline $\max . D_{x}(-)$ & 0.991 & $\mathbf{0 . 9 9 1}$ & 0.989 & $\mathbf{0 . 9 9 1}$ & 0.959 & $\mathbf{0 . 9 6 7}$ & 0.982 & 0.976 \\
\hline $\max . S N R E(\mathrm{~dB})$ & 26.76 & $\mathbf{3 2 . 8 8}$ & 31.09 & $\mathbf{3 1 . 8 3}$ & 26.70 & $\mathbf{3 2 . 9 8}$ & 30.34 & 30.81 \\
\hline $\min . A_{f}(\%)$ & 8 & $\mathbf{6}$ & 9 & $\mathbf{5}$ & 13 & $\mathbf{1 0}$ & 20 & 10 \\
\hline $\min . k_{(-)}$ & 1.35 & $\mathbf{2}$ & 1.1 & $\mathbf{1 . 4}$ & 1.35 & $\mathbf{4 . 5}$ & 1.4 & 1.4 \\
\hline $\min . \alpha_{v}(-)$ & 0.16 & $\mathbf{0 . 2 2}$ & 0.18 & $\mathbf{0 . 2}$ & - & - & - & - \\
\hline
\end{tabular}

Table 3: Evaluation of custom thresholding

\begin{tabular}{|l|c|c|c|c|}
\hline threshold level & \multicolumn{4}{|c|}{$V_{1}$} \\
\hline Mother wavelet / parameter & $\mathrm{db} 2$ & $\mathbf{d b 4}$ & $\mathrm{db} 6$ & dmey \\
\hline $\max . D_{x}(-)$ & 0.869 & $\mathbf{0 . 8 2 0}$ & 0.887 & $\mathbf{0 . 8 2 0}$ \\
\hline $\max . S N R E(\mathrm{~dB})$ & 26.88 & $\mathbf{3 5 . 7 2}$ & 29.38 & $\mathbf{3 2 . 2 3}$ \\
\hline $\min . A_{f}(\%)$ & 9 & $\mathbf{7}$ & 11 & $\mathbf{6}$ \\
\hline$k(-)$ & 1.35 & $\mathbf{2}$ & 1.1 & $\mathbf{1 . 4}$ \\
\hline$\alpha_{v}(-)$ & 0.16 & $\mathbf{0 . 2 2}$ & 0.18 & $\mathbf{0 . 2}$ \\
\hline$\tau_{v}(-)$ & 0.03 & $\mathbf{0 . 0 4}$ & 0.03 & $\mathbf{0 . 0 3}$ \\
\hline
\end{tabular}


standard deviation were used, and the best parameters were also searched for efficient ultrasonic signal noise reduction. The main idea of using group delay statistics is that the useful signal has a constant group delay in a certain frequency range. This frequency range depends on the frequency response of the ultrasonic transducer. The de-noising efficiency can be increased by using an appropriate window with frequency bandwidth and threshold level. In our evaluation only the Hamming window was used. In the case of threshold level and frequency bandwidth we changed the threshold level within $1-80 \%$ of the maximal amplitude of the Wiener filter transfer function and frequency bandwidth within 5-15 MHz. The Wiener filter was evaluated using the same parameter SNRE as in the case of DWT. An evaluation of the Wiener filter based group delay statistics is shown in Fig. 4. An ultrasonic signal with a different fault echo amplitude was also generated. The best results were obtained with a threshold level of $40 \%$ and a frequency bandwidth corresponding to $9 \mathrm{MHz}$. With this setting, the highest $S N R E=14.7 \mathrm{~dB}$. A comparison of the two algorithms shows that the $S N R E$ values for the Wiener filter based standard deviation are higher. However, the SNRE values are lower than in the case of DWT. This may be that because the Wiener filter is similar in shape to a band pass filter that suppresses only the frequencies outside the frequency range of the proposed filter.

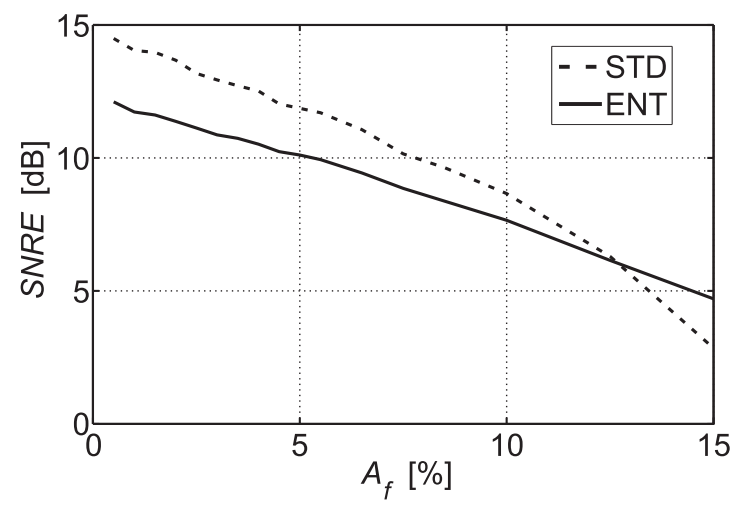

Fig. 4: Wiener filter evaluation

The last method applied here is blind source separation used on an ultrasonic signal and noise separation in the configuration shown in Fig. 1. Based on this configuration the ultrasonic signals were acquired. We obtained two ultrasonic signals that can be described using the following equations:

$$
\begin{aligned}
& x_{1}(t)=a_{1 s} s_{1 s}(t)+a_{1 n} n_{1 e}(t), \\
& x_{2}(t)=a_{2 s} s_{2 s}(t)+a_{2 n} n_{2 e}(t),
\end{aligned}
$$

where $s_{1 s}(t)$ and $s_{2 s}(t)$ is the source signal acquired with ultrasonic transducer no. 1 and no. 2, and $n_{e}(t)$ is electronic noise. The source signals in this configuration are considered to be all the reflections from the material structure (backscattering noise, fault echo and back-wall echo). If the basic presumptions of equation (6) are valid, sources $s_{1 s}(t)=s_{2 s}(t)$ and noise $n_{1 e}(t)=n_{2 e}(t)$. Here the presumptions are only theoretical, and if we investigate the real situation in detail the conditions are completely different. The ultrasonic waves propagated through the material structure from ultrasonic transducer no.
1 clearly have different reflections from the ultrasonic waves propagated from ultrasonic transducer no. 2. This means that the two sources are different, due to the different material structure, and it is clear that $s_{1 s}(t) \neq s_{2 s}(t)$. The situation with the noise $n_{e}(t)$ is the same. If the electronic noise structure from ultrasonic transducer no. 1 is to be equal to the electronic noise structure from transducer no. 2, the two ultrasonic transducers, the ultrasonic system and the cables and measurement conditions must have been completely the same. This is also impossible; nobody can design the same parts with the same noise characteristics, so $n_{1 e}(t) \neq n_{2 e}(t)$. From this simple overview it is clear that the basic presumptions cannot be fulfilled and the blind source separation method cannot be used in the area of ultrasonic non-destructive testing in the configuration presented here.

\section{Experimental results}

For the performance of all proposed de-noising algorithms, we used the ultrasonic signals acquired on samples of coarse-grained materials used for constructing aircraft engines. For all measurements, we used an ultrasonic transducer with a frequency of $25 \mathrm{MHz}$. The signals were measured above the flaw, and consequently de-noising algorithms were used. Fig. 5b shows the de-noised signal with DWT using the discrete Meyer mother wavelet, hard thresholding and a threshold based on standard deviation. The noise was effi-

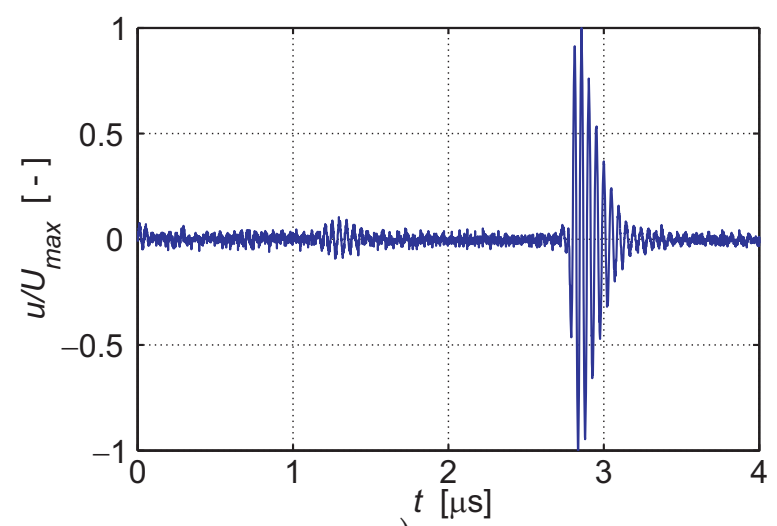

a)

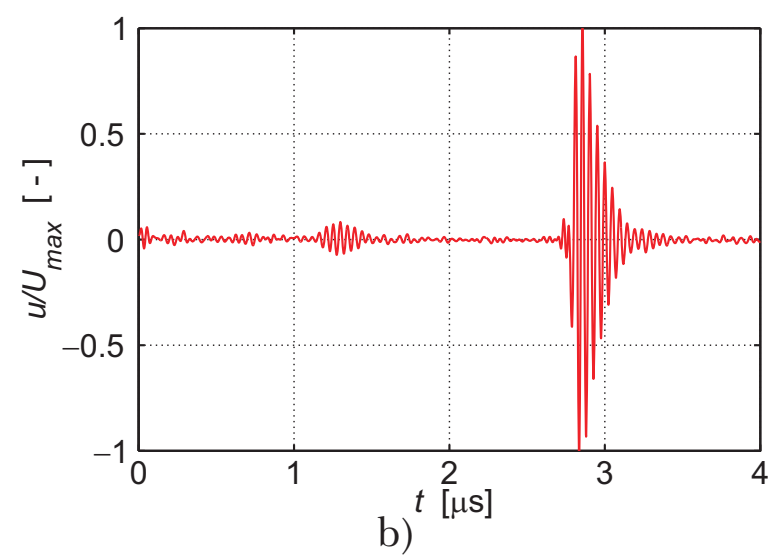

Fig. 5. Ultrasonic signal de-noising using the DWT algorithm, a) acquired signal, b) filtered signal 
ciently suppressed and the fault echo and back-wall echo are without amplitude changes.

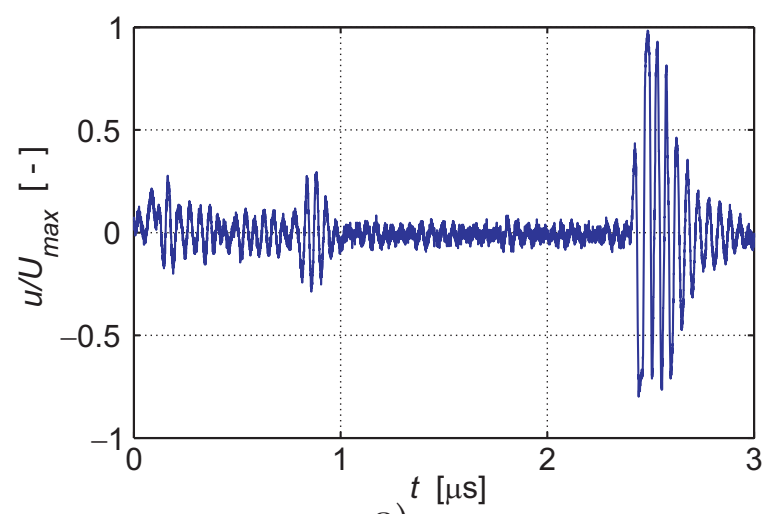

a)

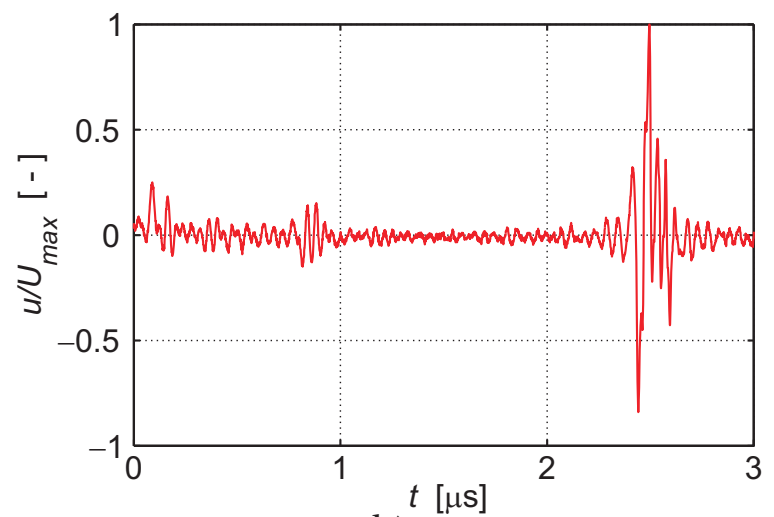

b)

Fig. 6. Ultrasonic signal de-noising using the Wiener filter, a) acquired signal, b) filtered signal

In the case of the Wiener filter, the noise was also efficiently suppressed, but the signal after de-noising is more corrupted by noise.

It can be seen that the noise was also efficiently suppressed but the fault echo amplitude was decreased. In general, it can be concluded that DWT is a more efficient method and more useful than the Wiener filter in the case of ultrasonic signal de-noising.

\section{Conclusion}

This paper describes and evaluates methods for ultrasonic signal de-noising and separation. Based on our analysis, the best de-noising method for efficient noise suppression is the discrete wavelet transform. The noise reduction for a signal with fault echo is $35 \mathrm{~dB}$. The amplitude of fault echo higher than $5 \%$ of the initial echo amplitude is without changes, and the fault echo can be easily detected. We also investigated improvements in fault detection sensitivity using the appropriate parameter setting. Our setting identifies the fault echo with amplitude comparable with the noise level. However, the Wiener filter using group delay statistics does not offer efficient noise suppression. The blind source separation method is not appropriate for separating the signal and the noise in ultrasonic signals, because the basic presumptions of this method are not fulfilled.

\section{Acknowledgments}

This research work has received support from research program No. MSM210000015 "Research of New Methods for Physical Quantities Measurement and Their Application in Instrumentation" of the Czech Technical University in Prague (sponsored by the Ministry of Education, Youth and Sports of the Czech Republic).

\section{References}

[1] Krautkrämer, J., Krautkrämer, H.: Ultrasonic Testing of Materials. Springer-Verlag, $4^{\text {th }}$ fully revised edition, 1990, 670 p., ISBN 3-540-51231-4.

[2] Drai, R., Sellidj, F., Khelil, M., Benchaala, A.: Elaboration of Some Signal Processing Algorithms in Ultrasonic Techniques: Application to Materials NDT. Ultrasonics, Elsevier, Vol. 38 (2000), p. 503-507.

[3] Louis, A. K., Maßß, P., Rieder, A.: Wavelets: Theory and Applications. John Wiley and Sons Ltd., England, 1997.

[4] Qi, Tian, Bilgutay, N. M. : Statistical Analysis of Split Spectrum Processing for Multiple Target Detection. IEEE Transaction on Ultrasonics, Ferroelectrics and Frequency Control, Vol. 45 (1998), No. 1, January 1998, p. 251-256.

[5] Zhenqing, Liu, Mingda, Lu, Moan, Wei: Structure Noise Reduction of Ultrasonic Signals Using Artificial Neural Network Adaptive Filtering. Ultrasonics, Elsevier: Vol. 35 (1997), p. 325-328.

[6] Shou-peng, Song, Pei-wen, Que: Wavelet Based Noise Suppression Technique and its Application to Ultrasonic Flaw Detection. Ultrasonics, Elsevier: Vol. 44 (2006), p. 188-193.

[7] Paul, S. A.: The Illustrated Wavelet Transform Handbook: Introductory Theory and Applications in Science, Engineering, Medicine and Finance. Napier University, Edinburgh, UK, 2004, ISBN 07-50-30692-0.

[8] Pardo, E., San Emeterio, J. L., Rodriguez, M. A., Ramos A.: Noise Reduction in Ultrasonic NDT Using Undecimated Wavelet Transforms. Ultrasonics, Elsevier: Vol. 44 (2006), p. 1063-1067.

[9] Xing, Li, Nihat, M. Bilgutay: Wiener Filter Realization for Target Detection Using Group Delay Statistics. IEEE Transaction on Signal Processing, Vol. 41 (1993), No. 6, June 1993.

[10] Izquierdo, M. A. G., Hernandez, M.G., Graullera, O., Ullate, L. G.: Time Frequency Wiener Filtering for Structural Noise Reduction. Ultrasonics, Elsevier, Vol. 41 (2002), p. 269-271.

[11] Gustafsson, M. G., Stepinski, T.: Studies of Split Spectrum Processing, Optimal Detection, and Maximum Likelihood Amplitude Estimation Using a Simple Clutter Model. Ultrasonics, Elsevier: Vol. 35 (1997), p. 31-52.

[12] Hyvarinen, A., Oja, E.: A Fast-fixed Point Algorithm for Independent Component Analysis. Neural computation, Vol. 9 (1997), p. 1483-1492. 
[13] Cichocki, A., Shun-ichi, A.: Adaptive Blind Signal and Image Processing: Learning Algorithms and Application. London: John Willey \& Sons, LTD., 2002, ISBN 0471-60791-6.

Ing. Václav Matz

e-mail:vmatz@email.cz

Doc. Ing. Marcel Kreidl, CSc.

phone: +420224352117

e-mail: kreidl@feld.cvut.cz

Ing. Radislav Šmíd, Ph.D.

phone: +420224352131

e-mail: smid@feld.cvut.cz

Department of Measurement

Czech Technical University in Prague

Faculty of Electrical Engineering

Technická 2

16627 Prague 6, Czech Republic 